\title{
Convection Effects on PGSE-NMR Self-Diffusion Measurements at Low Temperature: Investigation into Sources of Induced Convective Flows
}

\author{
Kee-Choo Chung, Hyo-Yeon Yu, and Sangdoo Ahn* \\ Department of Chemistry, Chung-Ang University, Seoul 156-756, Korea. E-mail: sangdoo@cau.ac.kr \\ Received April 22, 2011, Accepted May 3, 2011
}

\begin{abstract}
The effects of convection on the measurement of the diffusion coefficients of liquids by the pulsed gradient spin echo (PGSE) NMR method at low temperature are discussed. To examine the generation of convective flows, we used four different types of sample tubes in the diffusion measurements with temperature variation; a normal $5 \mathrm{~mm}$ NMR tube, a Shigemi tube, an ELISE type tube, and a capillary tube. Below room temperature, the calculated diffusion coefficients of chloroform in $5 \mathrm{~mm}$ o.d. type tubes increased with decreasing temperature, while those in the capillary tube decreased linearly. The convective flow was found to be significant even at low temperature and it seemed to be mainly induced by the transverse temperature gradient. It was also found that the capillary tube was most appropriate to measure the diffusion coefficients, since its small diameter is effective in suppressing the convective flows at both high and low temperatures.
\end{abstract}

Key Words : NMR, Diffusion, Convection, Capillary tube, Shigemi tube

\section{Introduction}

Various techniques to quantitatively determine the diffusion coefficients in liquids have been designed during the last few decades. ${ }^{1-4}$ Since the method using pulsed-gradientspin-echo (PGSE) NMR is easy to apply, it has been used in many fields, such as polymer science, ${ }^{5-7}$ soil science, ${ }^{8}$ and electrochemistry. ${ }^{9}$ However, some errors occur when measuring diffusion coefficients using PGSE-NMR techniques. In particular, at high temperatures far from ambient, the inhomogeneous heating of the sample generates vertical temperature gradients which induce strong convective flows. ${ }^{10-12}$ This additional thermal motion can cause the translational motion of the molecules to be faster than that induced by their intrinsic self-diffusion. Another source of error in diffusion measurements is the eddy currents. However, the effects due to eddy currents can be efficiently minimized by using satisfactory recovery delays and/or appropriate NMR pulse sequences, such as the bipolar gradient pulse longitudinal eddy-current delay (LED), ${ }^{13}$ which was also used in this experiment. Consequently, minimizing the convection of the liquid is the most important factor determining the precision of the diffusion coefficients obtained by PGSE NMR methods with sample heating. There have been many attempts to overcome this convection effect, including sample rotation, ${ }^{14}$ introducing a convection compensating pulse sequence, ${ }^{15}$ decreasing the sample size, ${ }^{16}$ increasing the sample viscosity, ${ }^{17}$ etc. The most frequently used approach to the problem of convection is the use of modified NMR tubes, ${ }^{17}$ such as capillary and/or Shigemi tubes.

The convection effects in low temperature PGSE diffusion experiments (needed air cooling) have not been as widely studied as those at high temperature, ${ }^{18}$ because it was thought that convective flow only occurred during the heat- ing of the sample. However, convection is also produced during cooling and can interfere with the determination of the self-diffusion coefficients at low temperatures. In this work, we discuss the convective flows in low temperature PGSE diffusion measurements and examine the generation and effects of convection depending on the structure of the sample tubes by comparing the experimental data obtained using a normal $5 \mathrm{~mm}$ tube, a Shigemi tube, an Experimental LIquid SEaling (ELISE) type tube ${ }^{19}$ and a capillary tube.

\section{Experimental}

All of the sample tubes were purchased from SigmaAldrich Co. The susceptibility of the coaxial insert of the Shigemi tube was matched to $\mathrm{CDCl}_{3}$. $\mathrm{CDCl}_{3}-d(99.8 \%)$ was also purchased from Sigma-Aldrich Co. and used without further purification. The NMR experiments for the determination of the diffusion coefficients were performed at various temperatures $(238-318 \mathrm{~K}$ ) on a Bruker Avance 600 spectrometer with bipolar pulse longitudinal eddy current delay pulse sequence (BPPLED). ${ }^{13}$ Spoil gradients were also applied at the diffusion period and the eddy current delay. Typically, a value of 1-2 ms was used for the gradient duration $(\delta), c a .70,100,130 \mathrm{~ms}$ for the diffusion time $(\Delta)$, and the gradient strength $(\mathrm{g})$ was varied from $c a .1 .5 \mathrm{G} / \mathrm{cm}$ to $70 \mathrm{G} / \mathrm{cm}$ in 16 steps. Each parameter was chosen to obtain $\sim 90 \%$ signal attenuation in the last step. The pulse repetition delay (including the acquisition time) between each scan was $10 \mathrm{sec}$ to avoid any contamination from inter-scan spin echoes. The time allowed for temperature stabilization after changing the target temperature was set to $30 \mathrm{~min}$ at least. Data acquisition and the calculation of the diffusion coefficients were performed using Bruker Topspin software. Under specific experimental conditions of temperature and $\Delta$, the diffusion coefficient was measured three times to 
evaluate the errors in the experiments. However, the variations were not significant in any of these cases (usually less than $1-2 \%$ ), thus the average values (without standard deviations) were used in this paper.

\section{Results and Discussion}

To examine the effects of the shape of the tube (e.g. its diameter and suppressor) on the convection of the liquid, four different types of sample tubes (a-d) were used to determine the diffusion coefficients $(D)$ of residual chloroform $\left(\mathrm{CHCl}_{3}\right)$ in $99.8 \% \mathrm{CDCl}_{3}$ solvent: (a) a normal $5 \mathrm{~mm}$ NMR tube, (b) a Shigemi tube, (c) an ELISE type $5 \mathrm{~mm}$ tube sealed with a second solvent $\left(\mathrm{D}_{2} \mathrm{O}\right)$ on top of $\mathrm{CDCl}_{3},{ }^{19}$ and (d) a capillary tube ( $2 \mathrm{~mm}$ o.d.) inserted in a normal $5 \mathrm{~mm}$ tube. The diffusion coefficients were obtained using three different diffusion times, $\Delta(70,100,130 \mathrm{~ms})$ at each temperature, as the results could be useful to describe the effects of convection on the molecular diffusion.

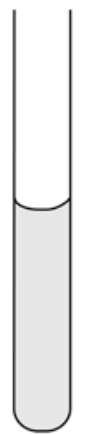

(a)

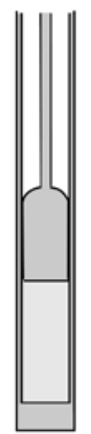

(b)

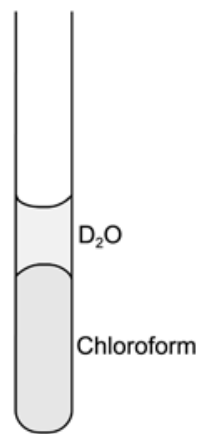

(c)

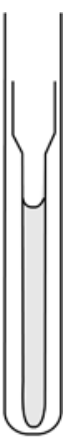

(d)
The diffusion coefficients of chloroform obtained at 238$318 \mathrm{~K}$ are shown in Table 1 and Figure 1 . In general, the self-diffusion coefficients $D$ should decrease as the temperature is decreased. However, the $D$ value versus temperature
Table 1. Calculated diffusion coefficients $\left(\mathrm{m}^{2} / \mathrm{sec}\right)$ at various temperatures and diffusion times $(\Delta)$

\begin{tabular}{|c|c|c|c|c|c|}
\hline Temp & $\Delta(\mathrm{ms})$ & $5 \mathrm{~mm}$ & Shigemi & ELISE & Capillary \\
\hline \multirow{3}{*}{238} & 70 & 3.79E-09 & 4.52E-09 & 7.57E-09 & $7.45 \mathrm{E}-10$ \\
\hline & 100 & 5.53E-09 & 5.87E-09 & $1.12 \mathrm{E}-08$ & $7.48 \mathrm{E}-10$ \\
\hline & 130 & 7.13E-09 & 7.16E-09 & $1.64 \mathrm{E}-08$ & $7.60 \mathrm{E}-10$ \\
\hline \multirow{3}{*}{248} & 70 & 2.44E-09 & 2.83E-09 & $7.45 \mathrm{E}-09$ & $8.53 \mathrm{E}-10$ \\
\hline & 100 & 3.17E-09 & $3.25 \mathrm{E}-09$ & $1.07 \mathrm{E}-08$ & $8.46 \mathrm{E}-10$ \\
\hline & 130 & 3.79E-09 & 4.89E-09 & $1.29 \mathrm{E}-08$ & $8.56 \mathrm{E}-10$ \\
\hline \multirow{3}{*}{258} & 70 & 2.07E-09 & 2.89E-09 & $6.90 \mathrm{E}-09$ & $1.00 \mathrm{E}-09$ \\
\hline & 100 & 2.43E-09 & $3.66 \mathrm{E}-09$ & $9.54 \mathrm{E}-09$ & $1.02 \mathrm{E}-09$ \\
\hline & 130 & 2.62E-09 & 4.40E-09 & $1.17 \mathrm{E}-08$ & $1.04 \mathrm{E}-09$ \\
\hline \multirow{3}{*}{268} & 70 & 2.42E-09 & 3.24E-09 & $6.45 \mathrm{E}-09$ & $1.25 \mathrm{E}-09$ \\
\hline & 100 & 2.88E-09 & 4.00E-09 & 8.97E-09 & $1.23 \mathrm{E}-09$ \\
\hline & 130 & 3.19E-09 & 4.62E-09 & $9.56 \mathrm{E}-09$ & $1.25 \mathrm{E}-09$ \\
\hline \multirow{3}{*}{278} & 70 & 2.48E-09 & 7.00E-09 & 4.84E-09 & $1.44 \mathrm{E}-09$ \\
\hline & 100 & $3.23 \mathrm{E}-09$ & $9.76 \mathrm{E}-09$ & $6.38 \mathrm{E}-09$ & $1.46 \mathrm{E}-09$ \\
\hline & 130 & 3.91E-09 & $1.27 \mathrm{E}-08$ & $8.06 \mathrm{E}-09$ & $1.44 \mathrm{E}-09$ \\
\hline \multirow{3}{*}{288} & 70 & $3.08 \mathrm{E}-09$ & 3.91E-09 & $3.24 \mathrm{E}-09$ & $1.83 \mathrm{E}-09$ \\
\hline & 100 & $3.88 \mathrm{E}-09$ & 4.71E-09 & $3.96 \mathrm{E}-09$ & $1.82 \mathrm{E}-09$ \\
\hline & 130 & 4.85E-09 & 4.90E-09 & 4.23E-09 & $1.81 \mathrm{E}-09$ \\
\hline \multirow{3}{*}{298} & 70 & 2.54E-09 & 2.43E-09 & $2.29 \mathrm{E}-09$ & $2.05 \mathrm{E}-09$ \\
\hline & 100 & 3.04E-09 & 2.75E-09 & 2.81E-09 & $2.04 \mathrm{E}-09$ \\
\hline & 130 & 4.06E-09 & $3.06 \mathrm{E}-09$ & 4.24E-09 & $2.04 \mathrm{E}-09$ \\
\hline \multirow{3}{*}{308} & 70 & 2.19E-08 & $1.52 \mathrm{E}-08$ & $1.95 \mathrm{E}-08$ & $2.32 \mathrm{E}-09$ \\
\hline & 100 & 3.19E-08 & $2.21 \mathrm{E}-08$ & $3.04 \mathrm{E}-08$ & $2.33 \mathrm{E}-09$ \\
\hline & 130 & 4.61E-08 & 3.07E-08 & $4.31 \mathrm{E}-08$ & $2.38 \mathrm{E}-09$ \\
\hline \multirow{3}{*}{318} & 70 & $6.54 \mathrm{E}-08$ & 3.83E-08 & $5.43 \mathrm{E}-08$ & $2.64 \mathrm{E}-09$ \\
\hline & 100 & $9.06 \mathrm{E}-08$ & $5.85 \mathrm{E}-08$ & $8.28 \mathrm{E}-08$ & $2.67 \mathrm{E}-09$ \\
\hline & 130 & 1.49E-07 & 8.89E-08 & $1.07 \mathrm{E}-07$ & 2.72E-09 \\
\hline
\end{tabular}

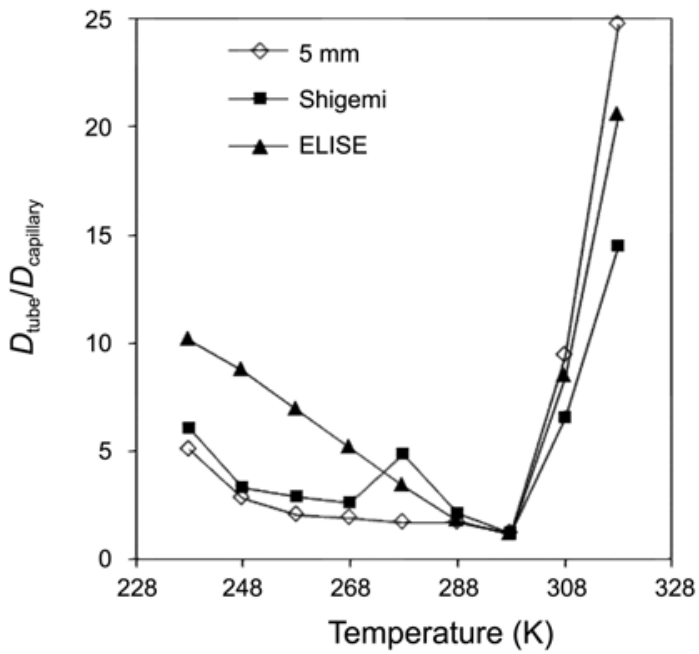

Figure 1. The temperature dependency of the calculated diffusion coefficients of chloroform (left) when the diffusion time $(\Delta)$ is $70 \mathrm{~ms}$. The $y$-axis scale is the common logarithmic type and the lines between the data points mean just linear interpolation. The error bars are omitted, since their sizes were smaller than those of the markers for all of the types of tubes. The ratios of the values from the $5 \mathrm{~mm}$ o.d. type tubes to that from the capillary tube (right) are also presented to allow the scales of the diffusion coefficients to be easily compared. 
graphs for the samples in the tubes with $5 \mathrm{~mm}$ o.d. (i.e. tubes $\mathrm{a}, \mathrm{b}$, and c) have $\mathrm{V}$-shapes with minimum values at $298 \mathrm{~K}$, while the diffusion coefficient for the capillary tube (d) decreases almost linearly with decreasing temperature according to the well-known Stoke-Einstein equation: ${ }^{13}$

$$
r_{H}=\frac{k T}{6 \pi \eta D}
$$

where $r_{H}=$ the hydrodynamic radius, $k=$ the Boltzmann constant, $T=$ the temperature, and $\eta=$ the viscosity of the solvent. Additionally, all of the $D$ values obtained from the 5 $\mathrm{mm}$ o.d. NMR tubes increased greatly with increasing diffusion time $(\Delta)$ and were much larger than the values obtained from tube $(d)$ at all temperatures (see Table 1). These observations strongly imply that there was a significant contribution of convection to the obtained $D$ values (except for those obtained from the capillary tube) in the case of cooling (below $298 \mathrm{~K}$ ) as well as heating (above $298 \mathrm{~K}$ ), in contrast to the general expectation.

The temperature regulation systems of most modern NMR apparatuses have a structure in which hot or cool air is injected from the bottom of the probes. Therefore, it is not surprising that convective flows arise in the liquid above room temperature, because the lower part of the sample tube is generally hotter than the upper part. This kind of convection is described as buoyancy-driven convection, which causes a large portion of the convection phenomena observed in our daily life. In the case of the $5 \mathrm{~mm}$ o.d. NMR tubes above $298 \mathrm{~K}$, buoyancy convection occurs and can lead to the significant movement of solvent molecules along the $z$ axis. This is observed in the form of the largely increased diffusion coefficient values compared with those obtained from the capillary tube. At $318 \mathrm{~K}$, as shown in Figure 1, the calculated diffusion coefficients of chloroform in the $5 \mathrm{~mm}$ o.d. NMR tubes were 15-25 times larger than those in the capillary tube.

On the other hand, below room temperature, there are variations in the temperature gradients along the $z$-axis, since cold air is supplied from the lower part of the sample tubes. This means that the bottom of the tube is cooler than the upper part, and the resulting inversed temperature gradient generally does not induce buoyancy-driven convection in the liquid. However, the experimental results at low temperature showed that some flow of the liquid occurs in addition to self-diffusive flow and, hence, the calculated diffusion coefficients of chloroform in the $5 \mathrm{~mm}$ o.d. NMR tubes were still much larger than those in the capillary tube (5-10 times at $238 \mathrm{~K})$. In order to explain these experimental results, the following two possibilities should be considered. First, the buoyancy-driven convection is still effective in the tube, in spite of the inversed temperature gradient. Secondly, there is another kind of liquid convection.

To examine the possibility of convection in the PGSENMR experiments at low temperature, it is necessary to carefully re-visit the variations of the temperature gradient in both the high- and low-temperature experiments. Figure 2 shows the expected patterns of the temperature
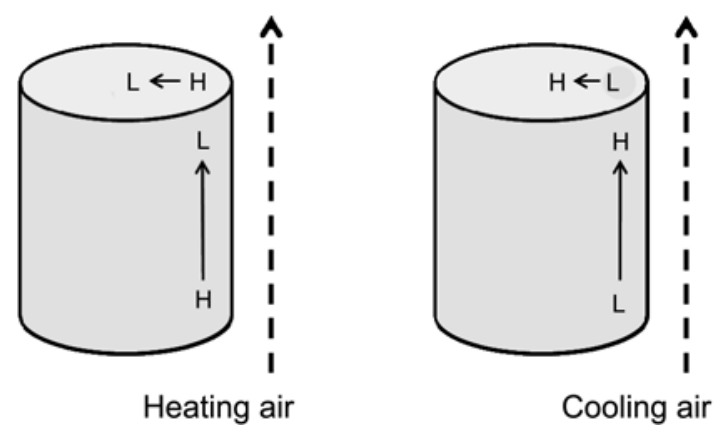

Figure 2. Variations of temperature gradients expected when hot or cold air is supplied from the bottom of the NMR tube, where $\mathrm{H}$ and $\mathrm{L}$ represent the high- and low-temperature components induced by the air flows, respectively.

gradient when hot or cold air is supplied from the bottom NMR tubes. As depicted, temperature gradients along both the longitudinal ( $z$-axis) and transverse $(x, y$-axis) directions could be created by the injection of hot or cold air. Loening and Keeler, using the chemical shift imaging method, reported the existence of the transverse temperature gradient of a liquid sample in an NMR tube at high temperature. ${ }^{20}$ Their result could be applied to low temperature experiments without critical argument. Consequently, the transverse temperature gradient combined with the longitudinal gradient would be able to induce a convective flow along oblique directions. This flow could be created at both high and low temperature. However, it might be just an additive effect at high temperature, whereas it would play a central role at low temperature.

The existence of the transverse temperature gradient may also introduce Marangoni convection as another kind of liquid convection. Marangoni convection refers to a flow which is produced by the variation of the surface tension. ${ }^{21}$ The inhomogeneity of the surface tension can be created by several factors including thermal gradients, hence Marangoni convection could be induced by the transverse temperature gradient on the liquid in the NMR tubes during high and/or low temperature experiments. The experimental results also seem to support the possibility of Marangoni convection. As shown in Figure 1, the calculated diffusion coefficients of chloroform in the Shigemi and ELISE tubes at high temperature had smaller values than those in a conventional $5 \mathrm{~mm}$ NMR tube, while the former values are larger than the latter at low temperature. If we introduce the Marangoni convection effects, these results can be explained as follows: the suppressors in the Shigemi and ELISE tubes (glass coaxial insert and water, respectively) are somewhat effective in suppressing the convection along the $z$-axis at high temperature, but they may induce a temperature gradient at the interface of chloroform, resulting in active Marangoni convection at low temperature. This might seem to be controversial in some ways, since Marangoni convection could be induced by the suppressors (located near the NMR active region) at either high or low temperature. The difference may come from the relative strengths of the two convective flows. At high temperature, the buoyancy-driven convection 

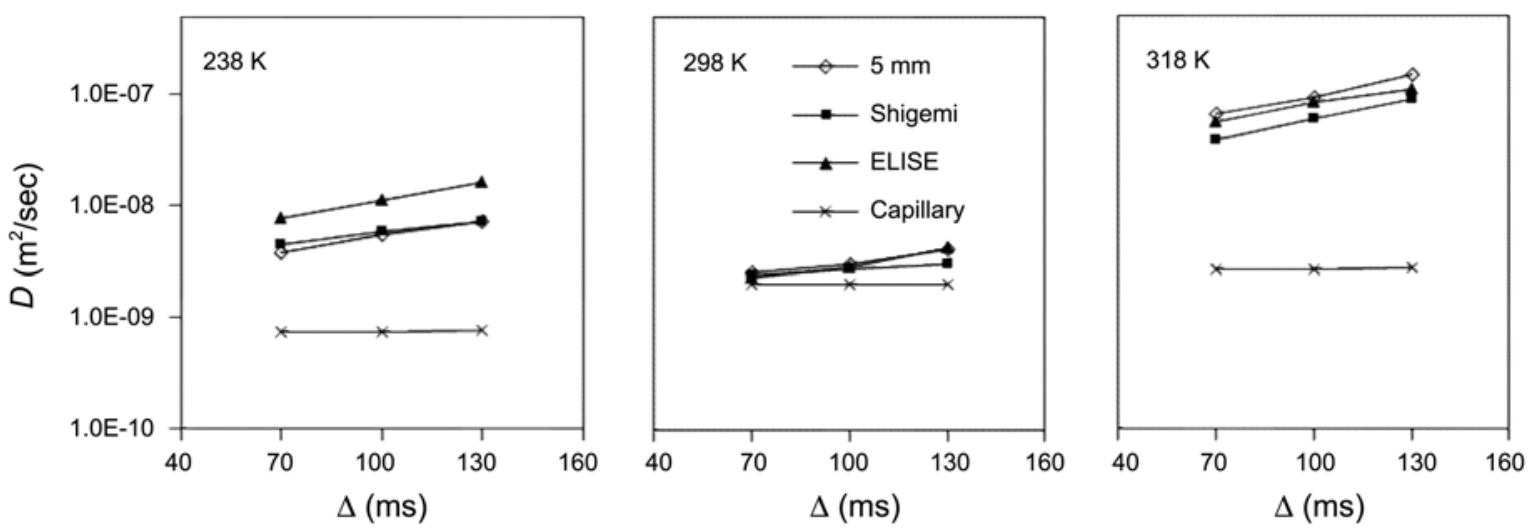

Figure 3. Comparison of the measured diffusion coefficients $(D)$ of chloroform at $238 \mathrm{~K}, 298 \mathrm{~K}$ and $318 \mathrm{~K}$ with varying diffusion time $(\Delta)$. The graphs correspond to the data in Table 1.

along the $z$-axis might be the critical effect, in which case the intrinsic role of the suppressors should be emphasized. Therefore, the solid suppressor (glass coaxial insert in the Shigemi tube) is more effective in suppressing the convection along the $z$-axis than the liquid one (water in the ELISE tube). On the other hand, as discussed earlier, the buoyancydriven convection is not significant at low temperature and, thus, the effect of Marangoni convection is revealed. It is likely that a lot of further research will be needed before we can describe quantitatively and conclusively the effects of the Marangoni convective flow at low temperature.

It was also found that the diffusion coefficients obtained from the ELISE type tube below $273 \mathrm{~K}$ was much larger than those obtained from the Shigemi and normal $5 \mathrm{~mm}$ tubes. This could be interpreted as being the result of the discrepancy in the thermal properties at the interface. Below $273 \mathrm{~K}$, the water suppressor of the ELISE tube turns into ice, whose thermal conductivity is much higher than those of chloroform and the glass insert of the Shigemi tube. The difference in the heat capacity is also bigger at the interface between ice and chloroform than between glass and chloroform. This discrepancy may create thermal turbulence at the interface (whether Marangoni convection is induced or not), which results in abnormally large diffusion coefficients being obtained. The ELISE NMR tube has an intrinsic advantage when it comes to sealing the target solvent completely with another solvent, since it is free from the bubbles which are usually observed in Shigemi tubes. However, it is found that this complete sealing effect is not that effective in suppressing the convective flows at either high or low temperature

The most effective way to overcome the convection effects for the purpose of determining the diffusion coefficients by PGSE NMR experiments would be to use a capillary tube. ${ }^{17,18}$ As shown in Figure 1, the temperature dependent diffusion coefficients of chloroform obtained from the capillary tube (d) seem to be very reliable, and to follow Eq. (1). The transverse (along $x, y$-axis) temperature gradient of the liquid in a capillary tube $(<2 \mathrm{~mm}$ i.d.) would be markedly reduced compared to that in a conventional $5 \mathrm{~mm}$ NMR tube, and this reduction might be sufficient to eliminate the additive flows, thus allowing for the determination of the diffusion coefficient at low temperature. However, at high temperature, where a temperature gradient exists along the $z$ axis regardless of the tube diameter, a more fundamental force may be needed to suppress the convection effectively. In a capillary tube, the increased surface-to-volume ratio due to the reduced tube diameter ensures that the role of the interfacial forces (created by the interactions between the fluid and glass wall), such as friction and adhesion, is dominant over that of the convective force. Hence, a capillary tube can sufficiently suppress the convection effects in PGSE NMR experiments at both high and low temperatures.

To evaluate the strength of the additive flows and the effects of the diffusion time $(\Delta)$ depending on the tube type, PGSE diffusion experiments were conducted with three different diffusion times $(\Delta=70,100,130 \mathrm{~ms})$. As can be seen in Figure 3, at high $(318 \mathrm{~K})$ and low $(238 \mathrm{~K})$ temperatures, the calculated diffusion coefficients increased greatly with increasing diffusion time, except for those from the capillary tube. (Note that the $y$-axis is a logarithmic scale.) For the same reasons as those discussed above, the variations of the calculated diffusion coefficients for the normal 5 $\mathrm{mm}$ tube having no suppressor were the greatest at high temperature $(318 \mathrm{~K})$, while those for the ELISE tube were the greatest at low temperature $(238 \mathrm{~K})$. As expected, the self-diffusion coefficients obtained from the $5 \mathrm{~mm}$ o.d. tubes (a-c) were closest to those from the capillary tube at nearambient temperature. Note that the ambient temperature was about 293-295 K during all of the experiments. As shown in Figure 3 , the observed differences in the diffusion coefficients at $298 \mathrm{~K}$ were also relatively small between $\Delta=70 \mathrm{~ms}$ and $130 \mathrm{~ms}$ compared with those at the other temperatures. However, the differences (20-80\%) were still sufficiently large to spoil the determination of the self-diffusion coefficient in the PGSE experiments at long $\Delta$ values, and this indicates that convection is effective even at near ambient temperatures if there is air flow and enough space to move ( $>>2 \mathrm{~mm}$ diameter). Among the $5 \mathrm{~mm}$ o.d. tubes, the Shigemi tube seems to be most effective in suppressing convective flows, at least near room temperature. 


\section{Conclusion}

We found that convection effects are significant in PGSE diffusion measurements with $5 \mathrm{~mm}$ o.d. NMR tubes, even at low temperatures, and that the transverse temperature gradient seems to be the main factor affecting the convective flow. The temperature dependence of the diffusion coefficients obtained under the influence of convective flows was strongly affected by the presence and type of compressors in the tubes. Marangoni convection should also be considered as a potential source of additional convective flows induced by the transverse temperature gradient.

Consequently, using a capillary tube seems to be the best way to eliminate convection from PGSE self-diffusion measurements, since it can minimize the transverse temperature gradient and enhance the interfacial forces preventing convective flows at both high and low temperatures.

Acknowledgments. This work was supported by the Chung-Ang University Research Grant in 2008 year.

\section{References}

1. Barson, C. A.; Dong, Y. M. Eur. Polymer J. 1990, 26, 329.

2. Das, H. A. J. Radioanal. Nucl. Chem. 2007, 273, 753.
3. Wakeham, W. A. Faraday Symp. Chem. Soc. 1980, 15, 145.

4. Yu, S. H.; Park, C. K.; Shin, C. B.; Cho, W. I. Bull. Korean Chem. Soc. 2011, 32, 852.

5. Logan, J. W.; Sherwood, M. H. Polymer Preprint 2005, 46, 413.

6. Kurkova, D.; Kriz, J.; Rodriguez-Cabello, J. C.; Arias, F. J. Polymer Int. 2007, 56, 186

7. Ahn, S.; Kim, E. H.; Lee, C. Bull. Korean Chem. Soc. 2005, 26, 331.

8. Staunton, S.; Nye, P. H. J. Soil Sci. 1987, 38, 651.

9. Suarez, S.; Chung, S. H.; Greenbaum, S.; Bajue, S.; Peled, E.; Duvdevani, T. Electrochim. Acta 2003, 48, 4.

10. Loening, N. M.; Keeler, J. J. Magn. Reson. 1999, 139, 334.

11. Jerschow, A. J. Magn. Reson. 2000, 145, 125.

12. Hedin, N.; Furó, I. J. Magn. Reson. 1998, 131, 126.

13. Johnson, C. S. Prog. Nucl. Magn. Reson. Spectrosc. 1999, 34, 203.

14. Lounila, J.; Oikarinen, K.; Ingman, P.; Jokisaari, J. J. Magn. Reson. A 1996, $118,50$.

15. Sorland, G. H.; Seland, J. G.; Krane, J.; Anthonsen, H. W. J. Magn. Reson. 2000, 142, 323.

16. Goux, W. J.; Verkruyse, L. A.; Saltert, S. J. J. Magn. Reson. 1990, $88,609$.

17. Hayamizu, K.; Price, W. S. J. Magn. Reson. 2004, 167, 328.

18. Martinínez-Viviente, E.; Pregosin, P. S. Helv. Chim. Acta 2003, 86, 2364.

19. Wieruszeski, J.-M.; Fritzinger, B.; Hanoulle, X.; Martins, J. C.; Lippens, G. J. Magn. Reson. 2008, 193, 37.

20. Loening, N. M.; Keeler, J. J. Magn. Reson. 2002, 159, 55.

21. Wilson, S. K. Acta Mechanica 1997, 124, 63. 